\title{
Interval Vibration Reduces Orthodontic Pain Via a Mechanism Involving Down-regulation of TRPV1 and CGRP
}

\author{
PEUNGCHALEOY THAMMANICHANON ${ }^{1}$, AUNWAYA KAEWPITAK ${ }^{2}$, \\ THUNWA BINLATEH ${ }^{3}$ and CHIDCHANOK LEETHANAKUL ${ }^{1}$ \\ ${ }^{1}$ Orthodontic Section, Department of Preventive Dentistry, \\ Faculty of Dentistry, Prince of Songkla University, Hat Yai, Thailand; \\ ${ }^{2}$ Department of Preventive Dentistry, Faculty of Dentistry, Prince of Songkla University, Hat Yai, Thailand; \\ ${ }^{3}$ Department of Physiology, Faculty of Science, Prince of Songkla University, Hat Yai, Thailand
}

\begin{abstract}
Background/Aim: The transient receptor potential vanilloid 1 (TRPVI) ion receptor is involved in the release of calcitonin gene-related peptide (CGRP), a major contributor to orthodontic pain. Approaches that attenuate expression of TRPVI and CGRP may reduce orthodontic pain. We explored the ability of high-frequency interval vibration to reduce orthodontic pain. Materials and Methods: Orthodontic force $(50 \mathrm{~g})$ was applied to both maxillary first molars in 8-weekold Wistar rats $(n=72)$. Vibration was applied at $125 \mathrm{~Hz}$ for $15 \mathrm{~min} /$ day. Duration of face grooming was assessed as a measure of orthodontic pain. Immunofluorescence and western blotting were used to assess TRPVI and CGRP in the trigeminal ganglia. Results: Compared to orthodontic force alone, application of vibration significantly decreased the duration of face grooming at $24 h$ and day 3 and reduced expression of TRPVI and CGRP at $24 \mathrm{~h}$. Conclusion: Vibration represents a promising mechanical approach to reduce orthodontic pain.
\end{abstract}

Orthodontic pain originates from orthodontic force-induced noninfectious inflammatory processes. Orthodontic force triggers the release of neuropeptides such as calcitonin generelated peptide (CGRP) and substance $\mathrm{P}$ (SP) from nociceptive fibers in the periodontal tissue. These neuropeptides promote inflammation in periodontal tissue

This article is freely accessible online.

Correspondence to: Chidchanok Leethanakul, Associate Professor, Prince of Songkla University, Orthodontic Section, Department of Preventive Dentistry, Faculty of Dentistry, Hat Yai, Songkhla, 90112 Thailand. Tel: +66 74287600, Fax: +66 74429875, e-mail: chidchanok.1@psu.ac.th or nokleethanakul@yahoo.com

Key Words: Orthodontic tooth movement, peptidergic nociceptors, trigeminal ganglia, TRPV1, vibration. and play an important role in evocation of orthodontic pain by transferring pain signals via sensory afferent neurons to neuronal bodies in the trigeminal ganglia (TG) and trigeminal nucleus caudalis, thereby generating pain perception within the sensory cortex (1-4). Various methods of relieving orthodontic pain have been investigated, including pharmacological (5), behavioral (6) and mechanical approaches (7-9). However, while some methods-such as administration of nonsteroidal antiinflammatory drugs-effectively reduce orthodontic pain, they also decrease the rate of tooth movement by inhibiting prostaglandin synthesis via inflammatory processes (10).

Mechanical vibration has been used as a supplementary approach to reduce musculoskeletal and dental pain $(11,12)$. Based on the theory of gate control, an appropriate vibration can activate mechanoreceptors, such as Meissner's corpuscle in subcutaneous and bone tissue, which will stimulate largediameter $A \beta$ nerve fibers to initiate inhibitory interneurons that, in turn, reduce pain perception by inhibiting nociceptive signals transmitted from projection neurons to the brain (13). In addition, there is evidence that vibration may act as analgesic by triggering retrograde transport within primary sensory neurons and subsequently reduce the release of nociceptive neuropeptides (14). However, the ability of vibration to relieve orthodontic pain has only been assessed in a limited number of studies.

Previous studies have established transient receptor potential vanilloid subtype 1 (TRPV1) as the primary nociceptive transducer involved in orthodontic pain. When triggered by noxious stimuli (15)-including orthodontic force-TRPV1 mediates the release of various neuropeptides $(16,17)$. Application of the TRPV1 agonist capsaicin has been reported to up-regulate calcitonin gene-related peptide (CGRP) in both the TG and trigeminal subnucleus caudalis in response to tooth movement (18), suggesting CGRP is a primary mediator released by TRPV1. CGRP is a member of the calcitonin family of peptides, which function as 
vasodilators and in transmission of nociceptive information. Up-regulation of CGRP is thought to increase the excitability of trigeminal neurons; CGRP is anterogradely transported to the periodontal tissues, resulting in amplification of orthodontic pain (4). Thus, TRPV1 and CGRP play important roles in the transmission of nociceptive information during orthodontic tooth movement.

The aims of this study were to assess the effects of highfrequency interval vibration on nocifensive behavior (the duration of face grooming) after application of orthodontic force using a rat model. To explore the related molecular mechanisms, we also examined the effects of orthodontic force with and without vibration on the expression of TRPV1 and CGRP in the neuronal cell bodies of the TG. These peptidergic neurons convey noxious information from the maxillary arch to the lateral restricted area of the maxillary division of the TG. We employed electrical toothbrushes to apply high-frequency vibration as these tools are inexpensive, simple to use, widely available and the approach is non-invasive $(9,19)$.

\section{Materials and Methods}

Experimental animals. Seventy-two 8-week-old male Wistar rats (Nomura Siam International Company, Bangkok, Thailand) weighing 200-250 g were used for this study. All animal procedures were approved by the Experimental Animal Center of Prince of Songkhla University (Songkhla, Thailand). Animals were housed in a strict hygienic conventional system in a temperature-controlled room in standard cages with food and water available ad libitum under a 12-h light cycle. The rats were allowed to acclimatize to the housing conditions for at least 1 week before the experiments were initiated.

The animals were randomly allocated to three groups: control group (Con), orthodontic force group (F), or orthodontic force combined with vibration group (FV). The eight rats in the control group received no interventions. The thirty-two rats in the $\mathrm{F}$ group received orthodontic treatment and the thirty-two rats in the FV group received orthodontic treatment and vibration. The groups and experimental timeline are shown in Figure 1A and B, respectively.

Experimental orthodontic tooth movement. To apply orthodontic force, the rats in the $\mathrm{F}$ and FV groups were anesthetized by intraperitoneal administration of $30 \mathrm{mg} / \mathrm{kg}$ ketamine and $10 \mathrm{mg} / \mathrm{kg}$ xylazine. A nickel-titanium close coil spring (Dentos, Dentaplex Daegu, Republic of Korea) was placed between the maxillary first molar and the maxillary incisor on both sides to move the maxillary first molars mesially, as previously described (20). The ligature wires were securely tied at the mesial surface of the maxillary first molar. Stainless steel 0.008-inch ligature wires were also placed around the maxillary incisors. A flowable light cure resin composite (Flow Tain, Reliance Orthodontic products Inc., Itasca, IL, USA) was then placed over the ligature wire on the buccal and palatal surfaces of the maxillary first molar and around the maxillary incisors. Finally, the coil-springs were activated and attached to the ligature wire, as illustrated in Figure 1C. The springs were activated to exert $50 \mathrm{~g}$ force in the $\mathrm{F}$ and $\mathrm{FV}$ groups.
Experimental application of vibration. A high-frequency vibration generator with an eccentric weight at the tip of an electric toothbrush was used to apply high-frequency vibration at $125 \mathrm{~Hz}$. The tip of the toothbrush was applied on the occlusal surface of the experimental first molars for $15 \mathrm{~min}$ immediately after insertion of the close coil-spring and once daily for $15 \mathrm{~min}$ on days 0,1 and 2 after insertion of the appliance. The timeline for the intervention is illustrated in Figure 1B.

Animal behavioral test. The face grooming test, a behavioral response to pain, provides a reproducible and reliable method of assessing orthodontic pain and was performed as previously described (21). The behavioral assay and video analysis were performed by a blinded investigator. The rats were placed in a $20 \times 10 \times 10 \mathrm{~cm}^{3}$ clear plastic box in a quiet environment for at least $15 \mathrm{~min}$ before the recordings were initiated. Digital video records were manually captured for $30 \mathrm{~min}$. The duration of grooming was averaged for 5 rats to yield the mean value for each group on days 1,3 and 7.

Immunofluorescent staining. TRPV1 and CGRP expression in the trigeminal ganglion were examined using an immunofluorescent assay (22). Rats ( $n=5$ rats per time-point per group) were terminally anesthetized by intraperitoneal administration of $30 \mathrm{mg} / \mathrm{kg}$ ketamine and $10 \mathrm{mg} / \mathrm{kg}$ xylazine at $12 \mathrm{~h}, 24 \mathrm{~h}$, day 3 , or on day 7 . The rats were transcardially perfused with $0.1 \mathrm{M}$ PBS followed by $4 \%$ paraformaldehyde (PFA). The trigeminal ganglia were dissected, post-fixed for 3 days in $4 \%$ PFA at $4^{\circ} \mathrm{C}$, cryoprotected by immersion in $0.1 \mathrm{M} \mathrm{PBS} / 30 \%$ sucrose at $4^{\circ} \mathrm{C}$ for 3 days and sectioned at $5 \mu \mathrm{m}$ using a cryostat.

Tissue sections were fixed in 4\% PFA for $40 \mathrm{~min}$, washed twice in PBS for $10 \mathrm{~min}$, incubated in blocking solution (10\% normal donkey serum in PBS with $0.25 \%$ Triton $\mathrm{X}-100$ ) for $60 \mathrm{~min}$ at room temperature, then incubated overnight at $4^{\circ} \mathrm{C}$ with polyclonal rabbit anti-TRPV1 (1:500; Abcam, Cambridge, MA, USA) or polyclonal rabbit anti-CGRP (1:500; Abcam). The antibodies were diluted in 5\% normal donkey serum in PBS with $0.25 \%$ Triton X-100 (PBST). After thoroughly washing three times in PBST for $10 \mathrm{~min}$, the sections were incubated with donkey anti-rabbit IgG-Fluorescein (FITC) secondary antibody (1:500; Jackson Immunoresearch, West Grove, PA, USA) for $2 \mathrm{~h}$ at room temperature. Sections were examined using a Zeiss fluorescence microscope (Carl Zeiss, Oberkochen, Germany). Control specimens were incubated with PBS without the primary antibodies. The fluorescence intensities in the maxillary branch of the TG were analyzed in the stored digital image sequences using Zen software (Carl Zeiss, Oberkochen, Germany). The control antibody-negative specimens were invariably negative.

Western blotting. Expression of TRPV1 and CGRP in the entire TG was examined by western blotting ( $n=3$ rats per time-point per group) at $12 \mathrm{~h}, 24 \mathrm{~h}$, day 3 and on day 7 . TG tissues were lysed in RIPA lysis buffer and the supernatants were stored at $-80^{\circ} \mathrm{C}$ until analysis. The total protein concentration of each sample was quantified using the BCA protein assay kit (Pierce ${ }^{\mathrm{TM}}$, Waltham, MA, USA). Lysates were separated on SDS-PAGE gels and the proteins were transferred onto polyvinylidene fluoride (PVDF) membranes and blocked with $5 \%$ skimmed milk in Tris-buffered saline with $0.1 \%$ Tween 20 (TBST) solution for $2 \mathrm{~h}$ at room temperature. The primary and secondary antibodies were polyclonal rabbit antiTRPV1 (1:500; Abcam), polyclonal rabbit anti-CGRP (1:500; 
A

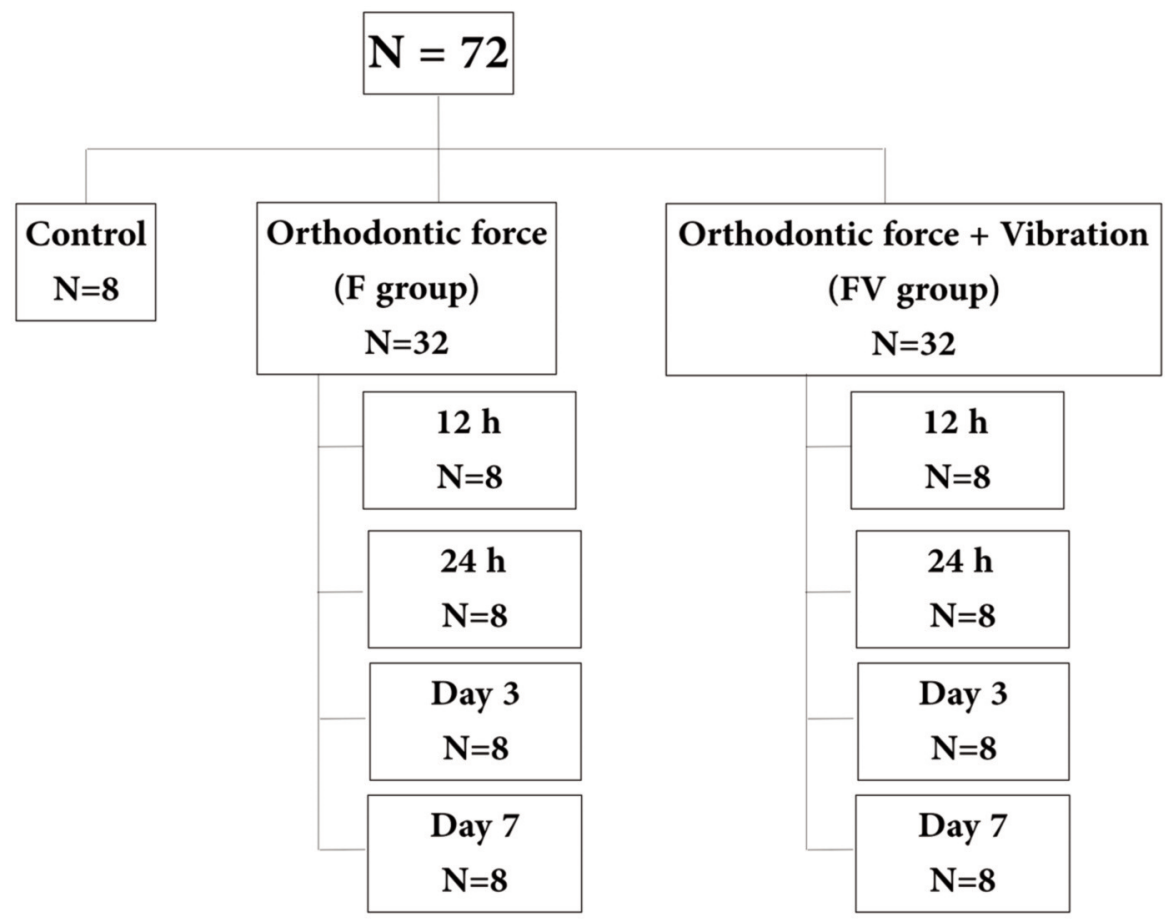

B


Figure 1. Schematic diagram of the experimental model. (A) The rats were divided into three groups: control group (Con; $n=8)$, orthodontic force group $(F ; n=32)$, and orthodontic force combined with vibration group $(F V ; n=32)$. The eight rats in the control group received no intervention. (B) Timeline of the experiment. (C) Coil springs were placed between the left and right maxillary first molars and incisors in the F and FV groups. The springs were activated to exert $50 \mathrm{~g}$ force. Vibration was applied for $15 \mathrm{~min}$ immediately after force insertion (day 0 ) and on day 1 and 2 . Rats were euthanized at 12 h, 24 h, on day 3 or 7 and the TG were examined by immunofluorescence staining and western blotting. 


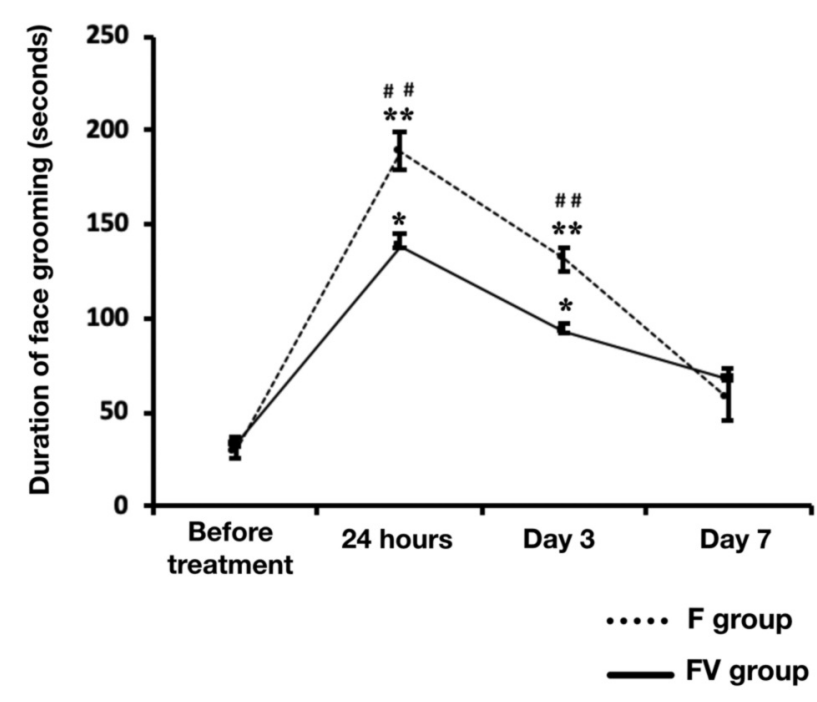

Figure 2. Orthodontic force induces spontaneous face grooming-evoked pain behaviors in rats. The pain scores were determined from the duration of face grooming activities at various time points after insertion of the coil-spring. The duration of face grooming in the $F$ and $F V$ groups is expressed relative to the control group; $* p<0.05$, ${ }^{*} p<0.001$ compared with the control group; \# $p<0.001$ for the comparison between the F group and FV group, Mann-Whitney U-test.

Abcam), polyclonal rabbit anti- $\beta$-actin (1:1000; Abcam) and secondary-goat anti-rabbit IgG (1:3000; Abcam) (23). The densities of the protein bands were analyzed using Image $\mathrm{J}(\mathrm{NIH}$, Bethesda, $\mathrm{MD}$, USA) (24) and normalized to the levels of the internal control housekeeping protein $\beta$-actin.

Statistical analysis. Data were analyzed using SPSS Statistics for Windows version 17.0 (SPSS Inc., Chicago, IL, USA) and are presented as mean \pm standard error of the mean. The KolmogorovSmirnov test was used to test normality of the data distributions. The Student's $t$-test was used to compare normally distributed data; the non-parametric Kruskal-Wallis and post-hoc Mann-Whitney $U$ tests were used to compare non-normally distributed data. The criterion for statistical significance was $p<0.05$.

\section{Results}

Interval vibration reduces the duration of pain behavior associated with application of orthodontic force in rats. The duration of face grooming was used as an indicator of orthodontic pain to determine whether application of vibration could reduce orthodontic pain during orthodontic tooth movement. The same five treatment-naive animals were used as the control group at each time point. As shown in Figure 2, the duration of face grooming was significantly greater at $24 \mathrm{~h}$ and day 3 after application of orthodontic force than in the untreated control animals. The duration of face grooming was significantly greater in the F group than that in the FV group at $24 \mathrm{~h}$ and day 3 . The duration of face grooming decreased in both the F and FV groups between $24 \mathrm{~h}$ and day 3 , but remained significantly higher in the $\mathrm{F}$ group compared to the FV group on day 3. The duration of face grooming decreased further between day 3 and day 7 and was similar in both groups on day 7 .

Vibration reduces the intensity TRPVI expression in the maxillary branch of the TG during orthodontic tooth movement. We determined the expression of TRPV1 in the maxillary branch of the TG to assess the mechanism by which vibration reduced orthodontic pain during tooth movement. As shown in Figure 3, the mean fluorescence intensity of TRPV1 was significantly higher in the F group compared to the control group at $24 \mathrm{~h} \quad(p<0.05)$ after application of orthodontic force; the mean fluorescence intensity (MFI) for TRPV1 peaked at $24 \mathrm{~h}$ in the F group. Furthermore, the MFI for TRPV1 was significantly higher in the $\mathrm{F}$ group than in the FV group at $24 \mathrm{~h}$, indicating that vibration reduced the induction of TRPV1 expression in response to orthodontic force. These findings imply that vibration may reduce orthodontic pain by suppressing TRPV1.

Vibration reduces TRPVI expression in the TG during orthodontic tooth movement. Western blotting of TG lysates was performed to confirm whether vibration decreased orthodontic pain during tooth movement by reducing the expression of TRPV1. Compared to the control group, application of orthodontic force significantly up-regulated TRPV 1 expression in the TG in the F group at $24 \mathrm{~h}$, then TRPV1 expression gradually decreased from days 3 and 7 . However, the FV group expressed significantly lower levels of TRPV1 than the F group at $24 \mathrm{~h}$ (Figure 4). These results indicate that application of vibration during tooth movement may reduce orthodontic pain by decreasing the expression of TRPV1 in the TG.

Vibration reduces CGRP expression in peptidergic neurons in the maxillary branch of the TG during orthodontic tooth movement. We quantified the expression of CGRP by immunofluorescent analysis to further examine the role of TRPV1 in response to the application of orthodontic force. As shown in Figure 5, compared to the control group, application of orthodontic force alone significantly increased the MFI for CGRP in the maxillary branch of the TG at 24 $\mathrm{h}$ and on day $3(p<0.05)$, with CGRP expression levels peaking at $24 \mathrm{~h}$ in the $\mathrm{F}$ group $(p<0.05)$. However, compared to the $\mathrm{F}$ group, application of vibration in combination with orthodontic force significantly reduced the MFI of CGRP in the TG in the FV group at $24 \mathrm{~h}$ and on day 3 . This indicates that vibration attenuated the up-regulation of CGRP induced by application of orthodontic force. 
A

Aptha

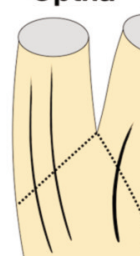

Trigeminal ganglion
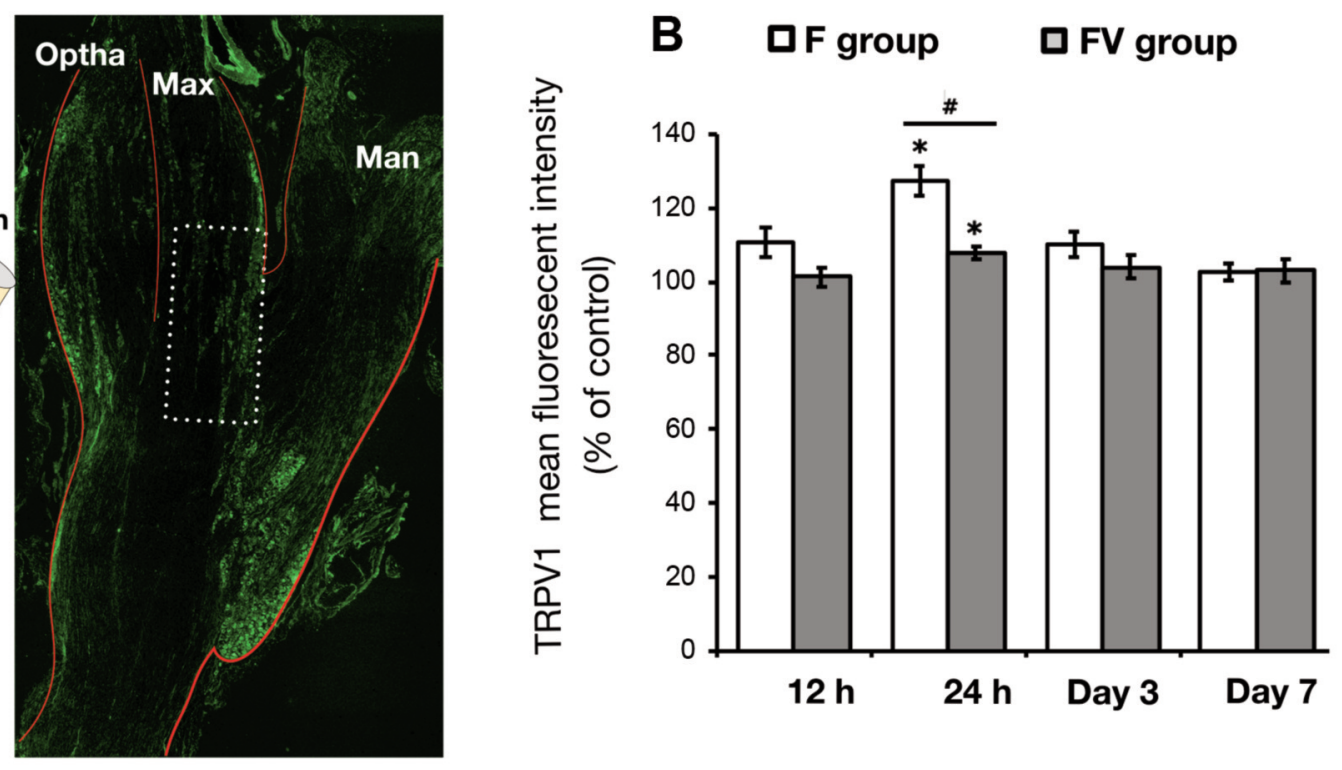

C

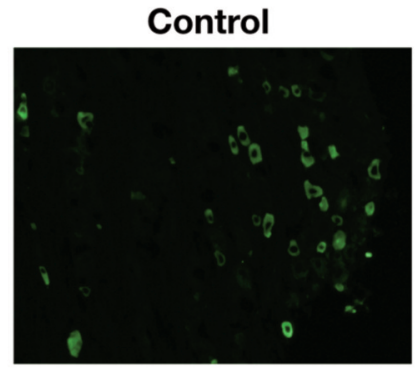

$12 \mathrm{~h}$
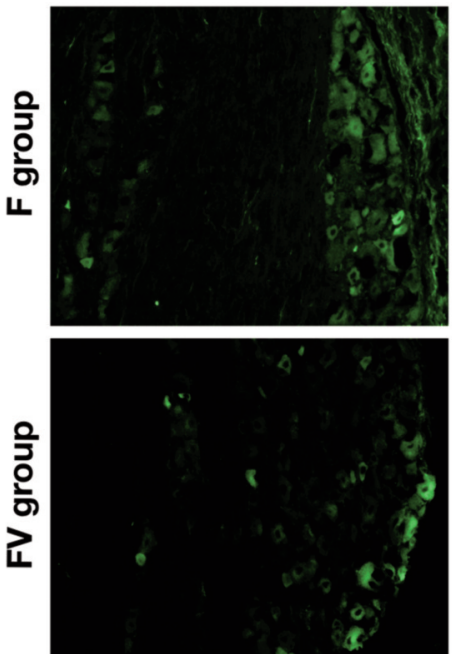

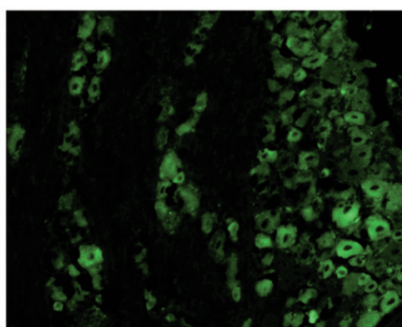

$24 \mathrm{~h}$

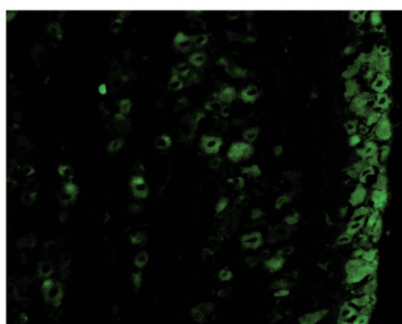

$B \quad \square F$ group $\square F V$ group

Negative control

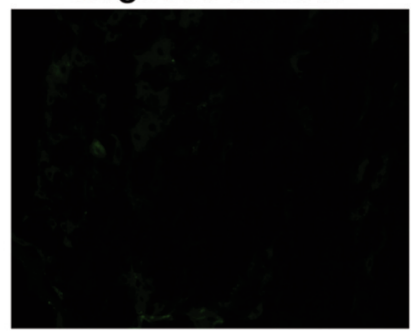

3 days

7 days
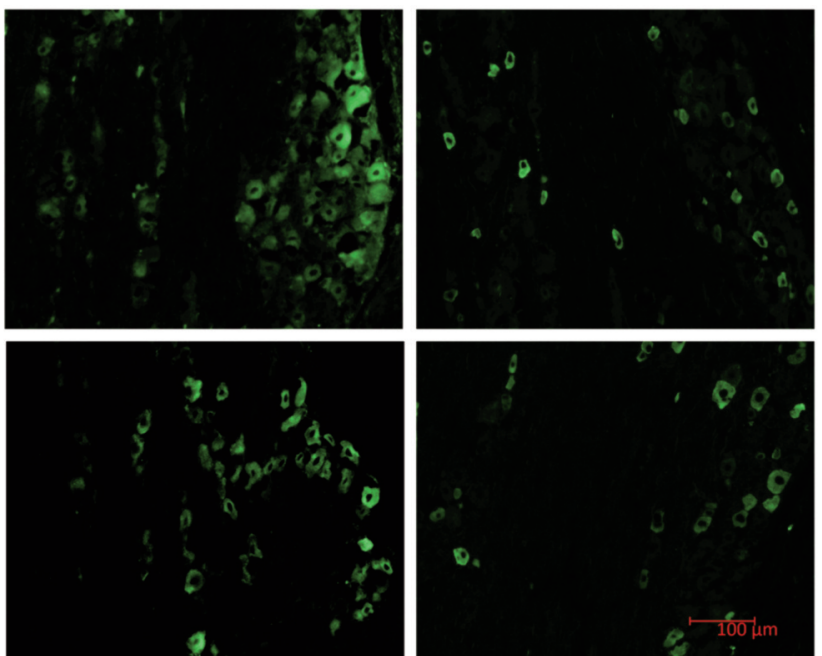

Figure 3. TRPV1 immunoreactivity in the maxillary branch of the TG during experimental tooth movement. (A) Representative images of immunofluorescent staining for TRPV1 in the maxillary branch area of the TG. TRPV1-positive cells are green. (B) Mean fluorescence intensity for TRPVI in the maxillary branch of the TG in the F and FV groups expressed relative to the control group. *p<0.05 compared with control group; ${ }^{\#} p<0.05$ for the comparison between the F group and FV group, Mann-Whitney U-test. 

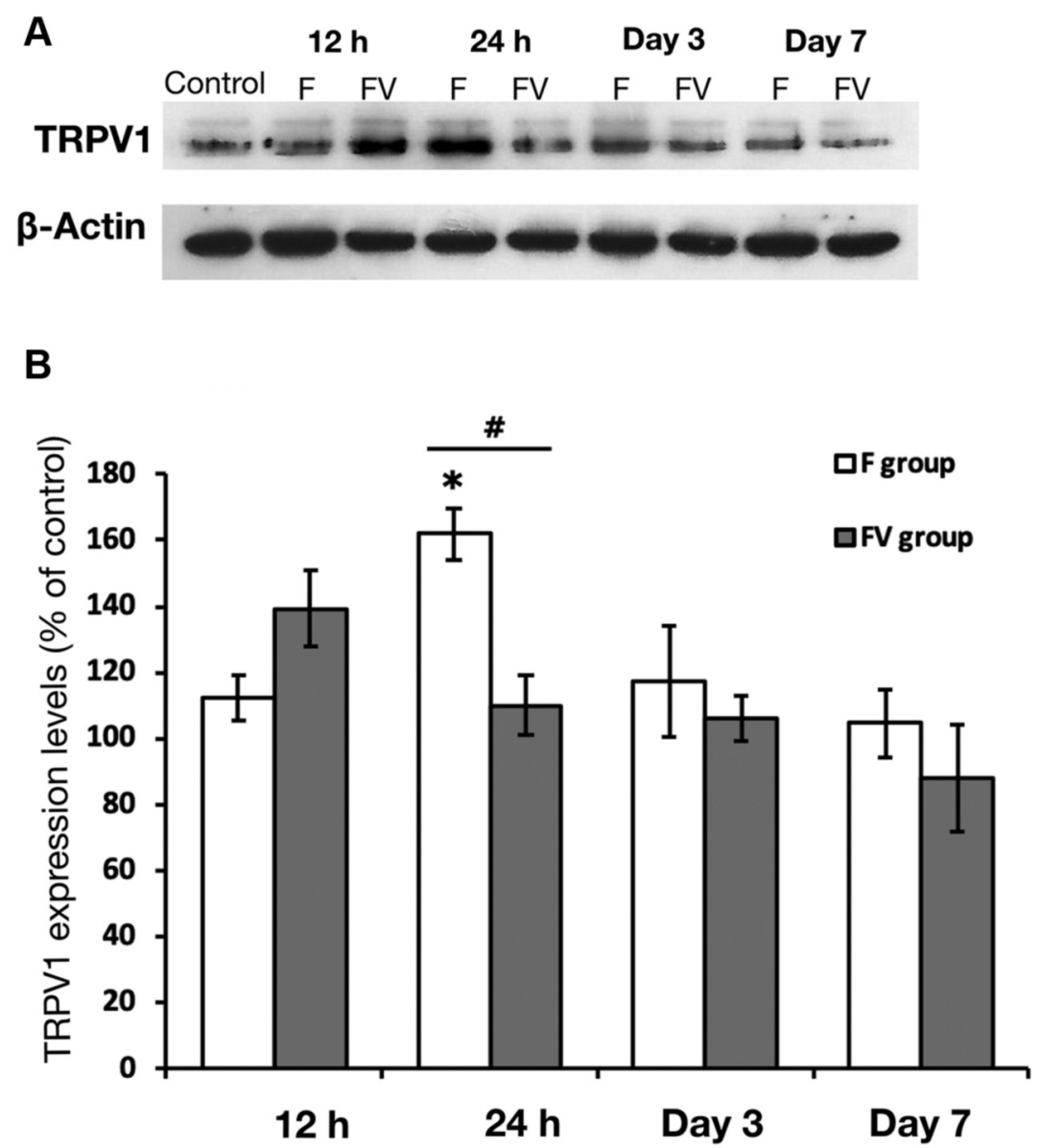

Figure 4. Vibration decreases TRPV1 expression in the TG during orthodontic tooth movement. (A) Representative western blots of TRPV1 protein expression in the TG of the F group and FV group at $12 \mathrm{~h}, 24 \mathrm{~h}, 3$ days and 7 days after application of orthodontic force. (B) Quantification of relative TRPVI expression in the TG at the different time points in the F group and FV group normalized to the control group. Data are mean \pm SEM $(n=3) ; * p<0.05$ compared with the control group; ${ }^{*} p<0.05$ for the comparison between the $F$ group and FV group, Mann-Whitney U-test.

Vibration reduces CGRP expression in the TG during orthodontic tooth movement. We performed western blotting in TG lysates to confirm the effects of high frequency vibration on CGRP expression during tooth movement. Compared to the control group, application of orthodontic force significantly up-regulated CGRP expression in the TG in the F group at 24 $\mathrm{h}$, then CGRP expression gradually decreased from day 3 to day 7. However, the FV group expressed significantly lower levels of CGRP than the F group at $24 \mathrm{~h}$ (Figure 6). These results indicate that applying vibration after application of orthodontic force reduced orthodontic pain by decreasing the expression of CGRP in the TG.

\section{Discussion}

We successfully established a rat model that produces measurable levels of orthodontic pain to investigate the ability of high-frequency interval vibration to reduce orthodontic pain. Our experimental model showed that applying vibration to the teeth after application of continuous orthodontic force decreased face grooming behavior and reduced the expression of CGRP and TRPV1 in the TG compared to rats subjected to orthodontic force alone.

Orthodontic pain is an undesirable side effect induced by orthodontic force during orthodontic treatment. Orthodontic force is received by the periodontal sensory nerve endings as a nociceptive stimulus. Pain signaling is subsequently transmitted to the TG, trigeminal nucleus caudalis and somatosensory cortex where orthodontic pain is perceived and a memory of unpleasant feelings is generated (3). In both humans and rat models, orthodontic pain develops $12 \mathrm{~h}$ after force application, peaks after 1 day, and then steadily decreases after 3-7 days. Similarly, the peak duration of face grooming behavior was observed $24 \mathrm{~h}$ after application of continuous orthodontic force, compared to the control group, and the 

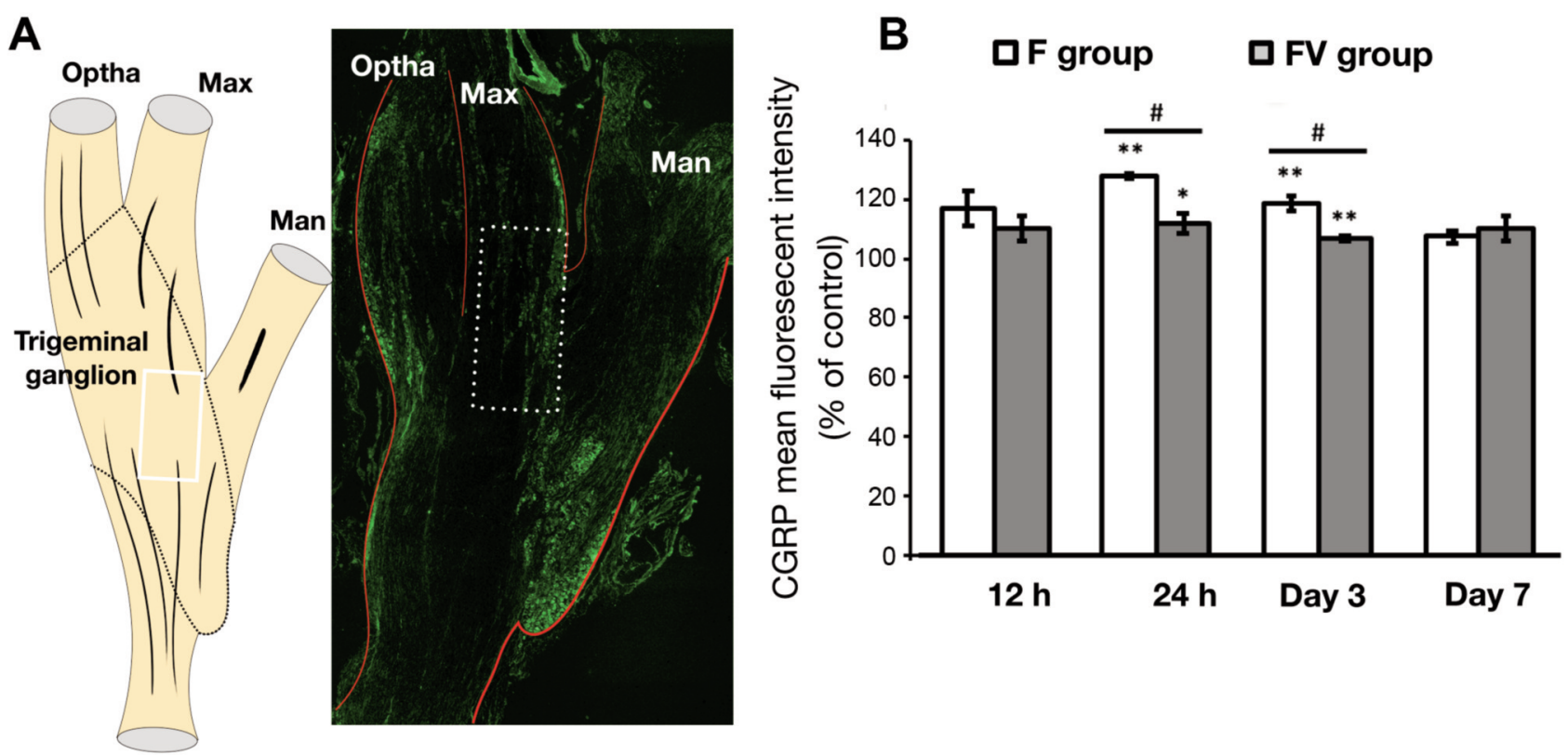

C
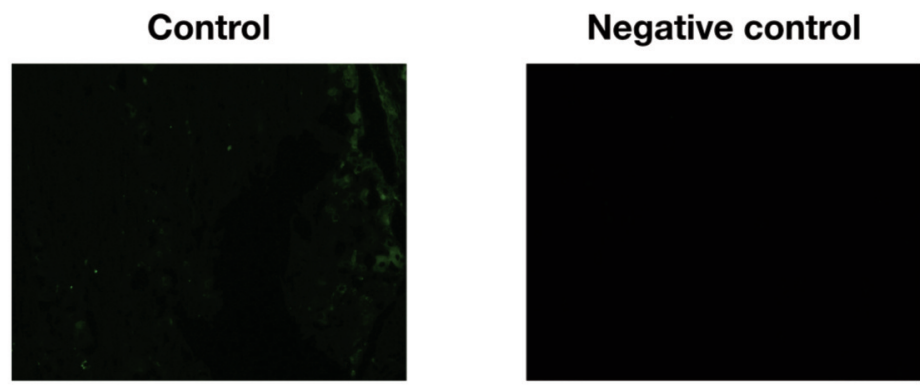

$12 \mathrm{~h}$
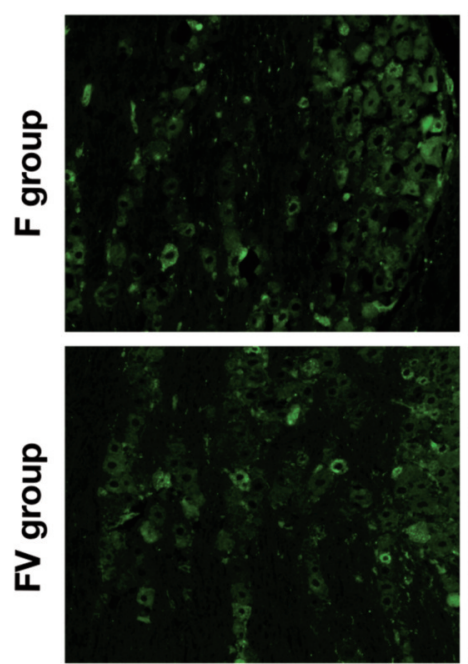

$24 \mathrm{~h}$
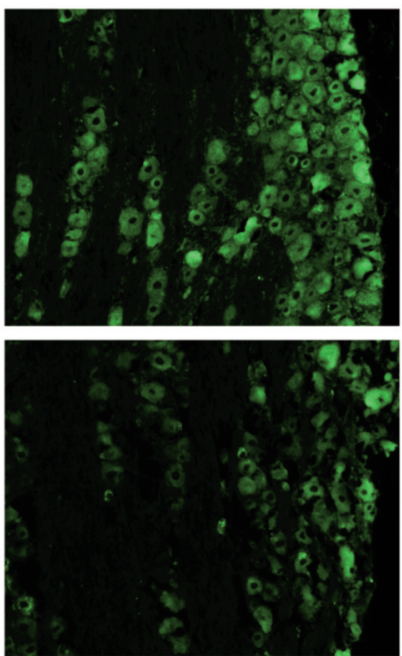

3 days
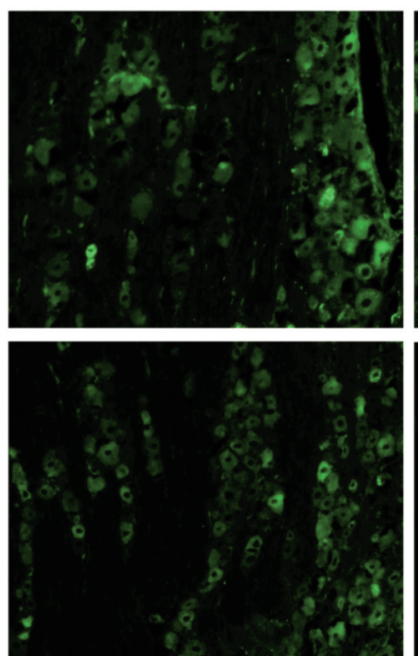

7 days
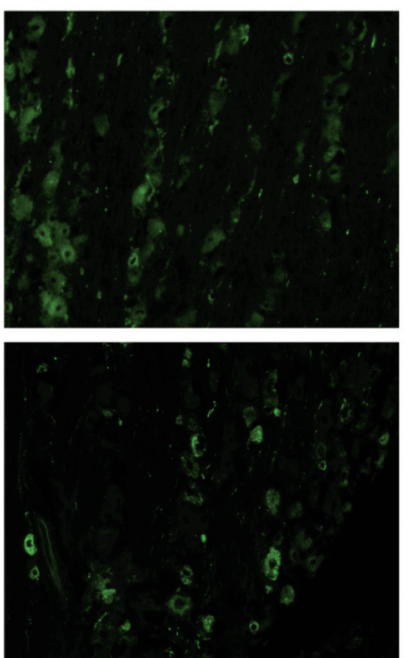

Figure 5. Vibration reduces the expression of CGRP in the TG during orthodontic tooth movement. (A) Representative images of immunofluorescent staining for CGRP in the maxillary branch area of the TG. CGRP-positive cells are green. (B) Mean fluorescence intensity for CGRP in the $F$ group and FV group expressed relative to the control group. Data are mean $\pm S E M(n=5) ; * p<0.05$, **p<0.001 compared with the control group; ${ }^{*}<0.05$ for the comparison between the F group and FV group, Mann-Whitney U-test. 

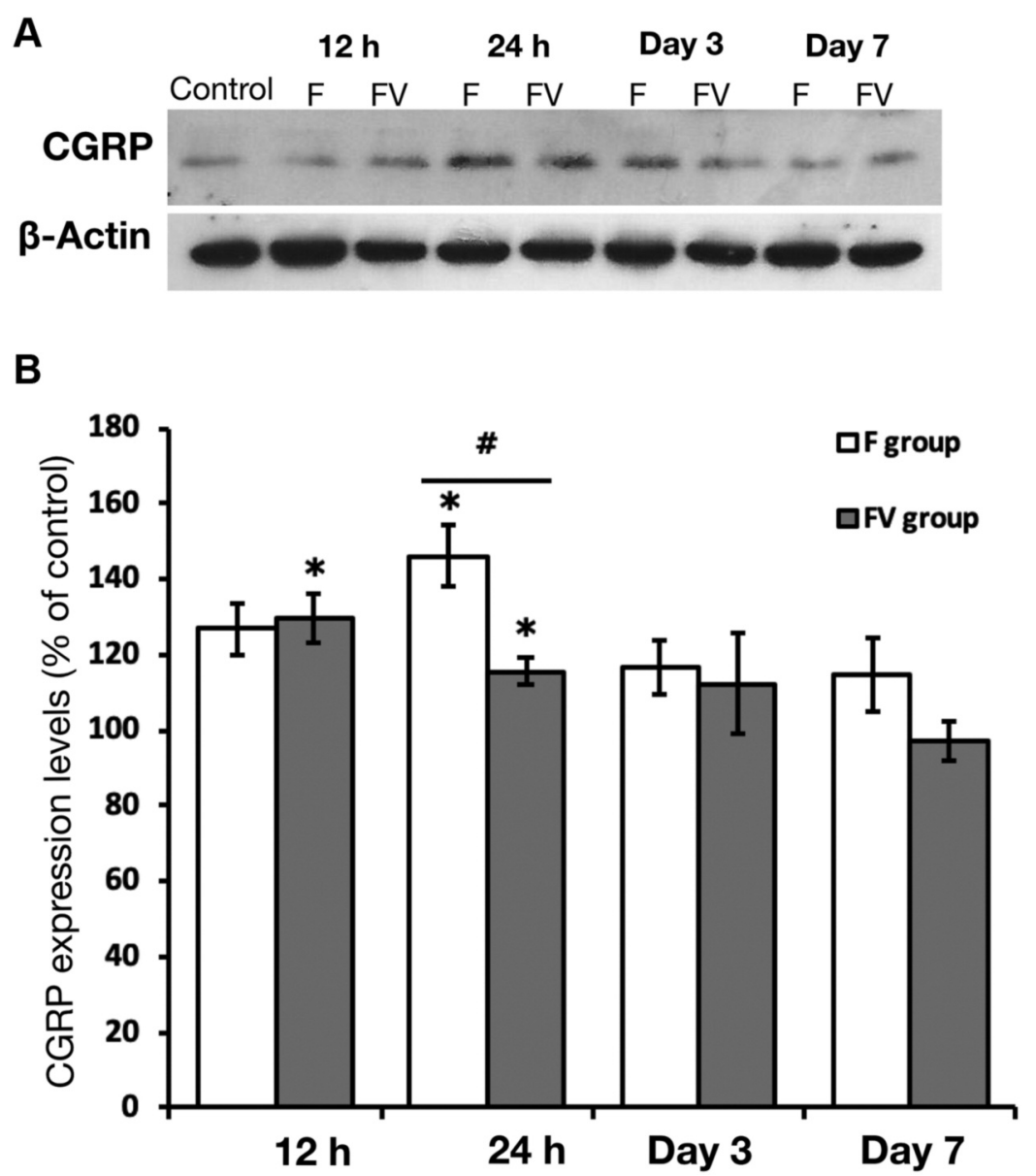

Figure 6. Vibration decreases CGRP expression in the TG during orthodontic tooth movement. (A) Representative western blots of CGRP protein expression in the TG of the F group and FV group at 12 h, 24 h, 3 days and 7 days after application of orthodontic force. (B) Quantification of relative CGRP expression in the TG at the different time points in the F group and FV group normalized to the control group. Data are mean \pm SEM $(n=3)$; * $p<0.05$ compared with the control group; ${ }^{*}<<0.05$ for the comparison between the $F$ group and FV group, Mann-Whitney U-test.

duration of face grooming behavior gradually reduced by day 7. Furthermore, applying vibration after application of continuous orthodontic force significantly decreased the duration of face grooming behavior, in agreement with the clinical study by Lobre et al. in 2015 (9). Likewise, in 2018, Alansari et al. (19) have reported that daily exposure to $5 \mathrm{~min}$ high-frequency vibration reduced pain perception in humans according to a numerical rating. Similarly, Stacy et al. (7) have demonstrated that exposure to $30 \mathrm{~Hz}$ vibration for $20 \mathrm{~min}$ reduced orthodontic pain, as indicated by a visual analog scale.

Two mechanisms may possibly explain the effect of mechanical vibration on orthodontic pain. First, according to gate control theory, application of vibration may reduce orthodontic pain by stimulating inhibitory interneurons, which would reduce the amount of pain signals transmitted by $A \delta$ and c fibers. Second, exposure to repeated vibration triggers axonal retrograde-transport in primary sensory neurons and subsequently decreases the release of nociceptive neuropeptides, and thereby reduces nociceptive transmission (14). Since some neuropeptides are potent peptide vasodilators, vibration-induced reductions in the levels of nociceptive neuropeptides may also decrease the area of periodontal ligament compressed by temporary displacement of the teeth-including nerve fibers and occluded blood vessels-and thus promote blood flow (25).

Furthermore, our data showed that TRPV1 and CGRP were up-regulated in the TG by orthodontic force. However, applying vibration after application of orthodontic force remarkably reduced TRPV1 and CGRP expression. TRPV1 is a polymodal TRP receptor channel that can be activated by noxious temperatures, changes in $\mathrm{pH}$ or voltage, and fatty acid amides and vanilloids. TRPV1 is considered to be an interesting nociceptive target for relieving pain, including orthodontic pain (26). A previous study has found TRPV1 


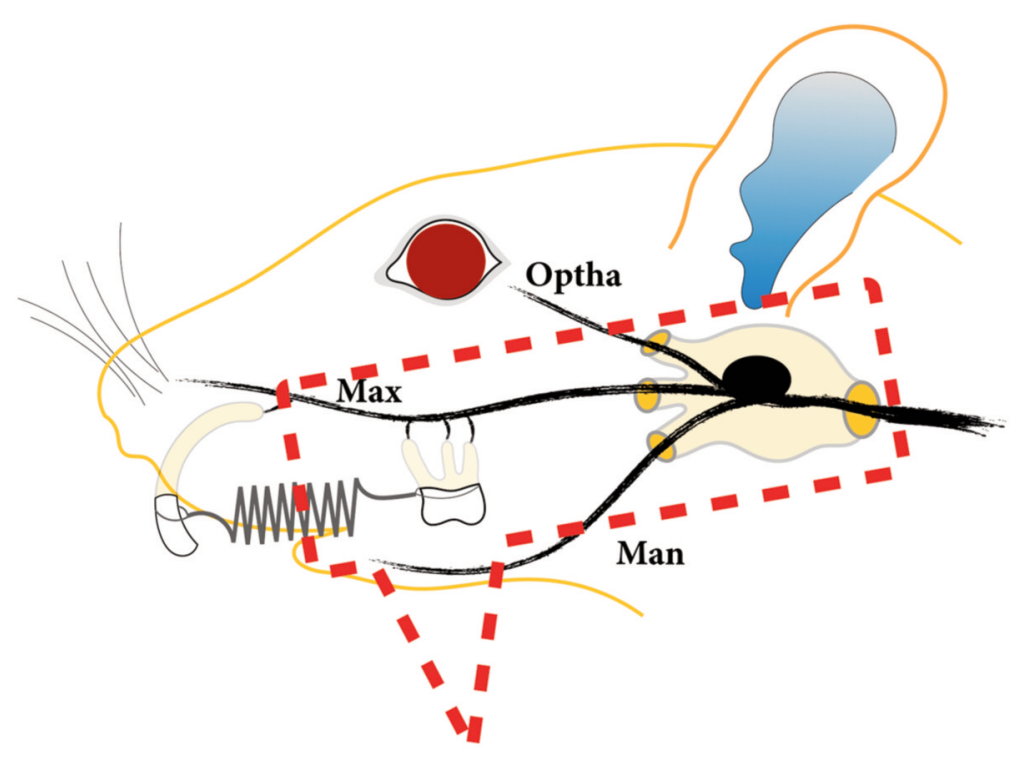

\section{F group}

TG

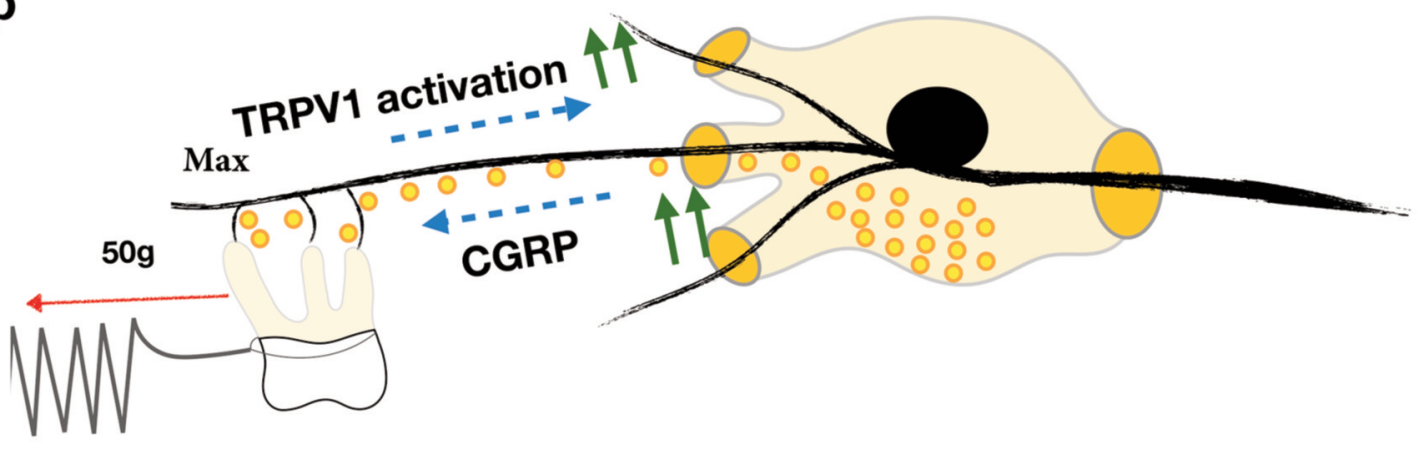

\section{TG}

\section{FV group}

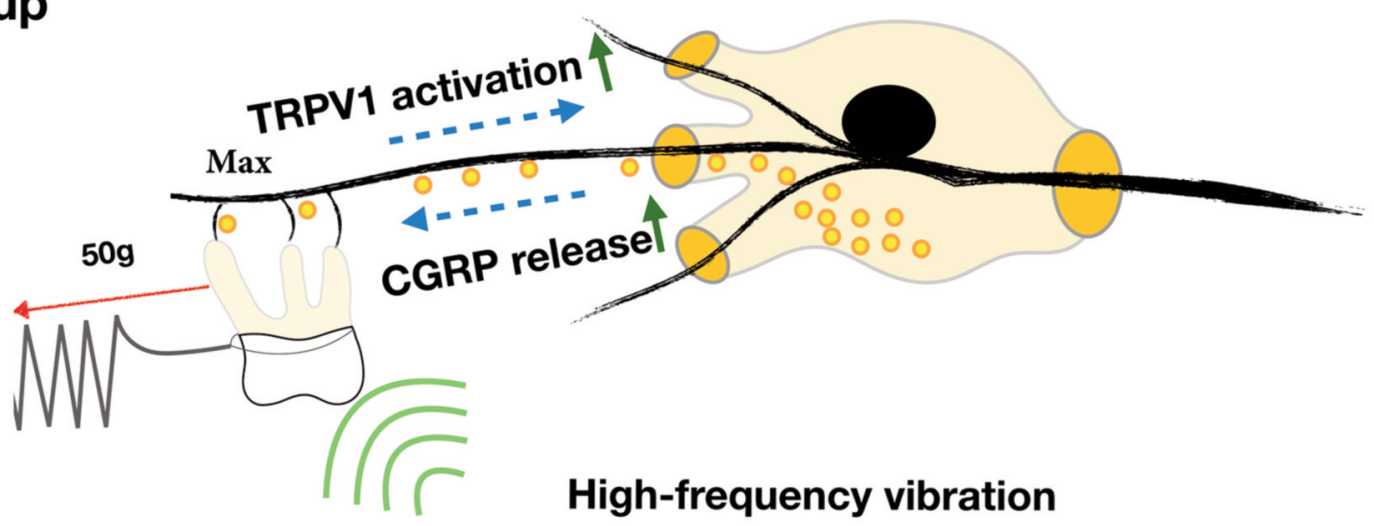

Figure 7. Schematic diagram of the proposed mechanisms by which high frequency vibration decreases TRPV1 and CGRP expression in the TG during orthodontic tooth movement. 
mRNA and protein expression were elevated in the TG during orthodontic pain. Moreover, orthodontic force stimulates TRPV1 sensory receptors in the nerve endings of TG neurons in periodontal tissues and subsequently increases calcium influx into the cell, thereby promoting the release of neuropeptides, including CGRP. CGRP is a neuropeptide responsible for nociceptive transmission that can induce neurogenic inflammation associated with dental pain (27). Upregulation of CGRP increases the excitability of trigeminal neurons and, additionally, CGRP is anterogradely transported to periodontal tissues, which amplifies orthodontic pain (4). A previous study has demonstrated a relationship between TRPV1 and CGPR by applying the TRPV1 agonist capsaicin during tooth movement. The TRPV1 agonist up-regulated CGRP in the TG and trigeminal subnucleus caudalis (18). Collectively, the decreases in TRPV1 and CGRP expression observed in the TG after applying vibration during orthodontic force-induced experimental tooth movement mirror the reductions in orthodontic pain conferred by vibration. Thus, our data support the roles of TRPV1 and CGRP in the transmission of nociceptive information during orthodontic tooth movement.

In addition, the duration of face grooming was significantly lower in the FV group compared to the F group at $24 \mathrm{~h}$ and on day 3 . In contrast, western blotting only showed significant decreases in TRPV1 and CGRP in both groups at $24 \mathrm{~h}$. One possible explanation is that CGRP stimulated the production of inflammatory cytokines that amplified pain behavior on day 3 , in addition to the direct effects of CGRP. Moreover, immunofluorescent staining showed that CGRP was expressed at significantly higher levels in the F group than in the control group at $24 \mathrm{~h}$ and 3 days, while TRPV1 expression was significantly increased only at $24 \mathrm{~h}$. Therefore, the sustained increase in CGRP expression compared to TRPV1 could possibly be due to a positive feedback loop, in which released CGRP causes local vasodilation and inflammatory accumulation, which in turn trigger the release of more $\operatorname{CGRP}(4,28)$. Notably, immunofluorescent staining revealed CGRP expression was significantly lower in the FV group compared to the F group at $24 \mathrm{~h}$ and 3 days. In contrast, CGRP expression was only significantly different between both groups at $24 \mathrm{~h}$ in western blot analysis. One possible explanation is that the entire TG, including the mandibular branch and ophthalmic branch were assessed in western blot analysis, whereas the maxillary branch-which directly receives pain signaling from maxillary first molar teeth-was only examined by immunofluorescence.

In summary, we report vibration has the potential to reduce orthodontic pain, possibly via mechanisms linked to reduced expression of TRPV1 and CGRP (Figure 7). These findings may provide a novel therapeutic approach to relieve orthodontic pain based on vibrational analgesia.

\section{Funding}

The Authors received a grant from the Graduate School, Prince of Songkla University for Scholarship Awards for Thai Ph.D. Students under Thailand's Education Hub for Southern Region of ASEAN Countries [TEH 2560-004].

\section{Conflicts of Interest}

The Authors declare that there are no conflicts of interest regarding this study.

\section{Authors' Contributions}

Experiment design, P.T., A.K. and C.L.; Experimental work, P.T., T.B.; Validation and analysis of data, P.T., T.B. and C.L.; Writing original draft, P.T., A.K., T.B. and C.L.

\section{Acknowledgements}

The Authors would like to thank Prof. Dr. Prasit Pavasant and Asst. Prof. Dr. Pilaiwanwadee Hutamekalin for providing critical comments. The Department of Pathology, Faculty of Medicine, Prince of Songkla University and Oral Neuroscience and Molecular Biology of Dental Pulp and Bone Cell Research Unit and the Research Center of the Faculty of Dentistry, Prince of Songkla University are acknowledged for use of their research facilities.

\section{References}

1 Krishnan V: Orthodontic pain: from causes to management-a review. Eur J Orthod 29(2): 170-179, 2007. PMID: 17488999.

2 Masella RS and Meister M: Current concepts in the biology of orthodontic tooth movement. Am J Orthod Dentofacial Orthop 129(4): 458-468, 2006. PMID: 16627170.

3 Long H, Wang Y, Jian F, Liao LN, Yang X and Lai WL: Current advances in orthodontic pain. Int J Oral Sci 8(2): 67-75, 2016. DOI: $10.1038 /$ ijos.2016.24

4 Long H, Liao L, Gao M, Ma W, Zhou Y, Jian F: Periodontal CGRP contributes to orofacial pain following experimental tooth movement in rats. Neuropeptides 52: 31-37, 2015. PMID: 26164378. DOI: 10.1016/j.npep.2015.06.006

5 Gupta M, Kandula S, Laxmikanth SM, Vyavahare SS, Reddy SB and Ramachandra CS: Controlling pain during orthodontic fixed appliance therapy with non-steroidal anti-inflammatory drugs (NSAID): a randomized, double-blinded, placebo-controlled study. J Orofac Orthop 75(6): 471-476, 2014. PMID: 25355194. DOI: $10.1007 / \mathrm{s} 00056-014-0243-7$

6 Wang J, Wu D, Shen Y, Zhang Y, Xu Y and Tang X: Cognitive behavioral therapy eases orthodontic pain: EEG states and functional connectivity analysis. Oral Dis 21(5): 572-582, 2015. PMID: 25644503. DOI: 10.1111/odi.12314

7 Marie S, Powers M and Sheridan J: Vibratory stimulation as a method of reducing pain after orthodontic appliance adjustment. J Clin Orthod 37(4): 205-208, 2003. PMID: 12747073.

8 Woodhouse NR, DiBiase AT, Papageorgiou SN, Johnson N, Slipper C and Grant J: Supplemental vibrational force does not reduce pain experience during initial alignment with fixed 
orthodontic appliances: a multicenter randomized clinical trial. Sci Rep 5: 17224, 2015. PMID: 26610843. DOI: 10.1038/srep17224

9 Lobre WD, Callegari BJ, Gardner G, Marsh CM, Bush AC and Dunn WJ: Pain control in orthodontics using a micropulse vibration device: A randomized clinical trial. Angle Orthod 86(4): 625-630, 2015. PMID: 26496680. DOI: 10.2319/072115-492.1

10 Fang J, Li Y, Zhang K, Zhao Z and Mei L: Escaping the adverse impacts of NSAIDs on tooth movement during orthodontics: current evidence based on a meta-analysis. Medicine 95(16): 3256, 2016. PMID: 27100413. DOI: 10.1097/MD.0000000000003256

11 Ottoson D, Ekblom A and Hansson P: Vibratory stimulation for the relief of pain of dental origin. Pain 10(1): 37-45, 1981. DOI: 10.1016/0304-3959(81)90043-9

12 Lundeberg T, Nordemar R and Ottoson D: Pain alleviation by vibratory stimulation. Pain 20(1): 25-44, 1984. PMID: 6333660. DOI: $10.1016 / 0304-3959(84) 90808-x$

13 Basbaum AI, Bautista DM, Scherrer G and Julius D: Cellular and molecular mechanisms of pain. Cell 139(2): 267-284, 2009. PMID: 19837031. DOI: 10.1016/j.cell.2009.09.028

14 Pavel J, Hricová Lu, Jergová S and Lukáčová N: The impact of short-lasting repeated vibrations on retrograde axonal transport, the expression of CGRP and parvalbumin in lower lumbar dorsal root ganglia. Brain Res 1396: 1-10, 2011. PMID: 21561603. DOI: $10.1016 /$ j.brainres.2011.04.023

15 Premkumar LS and Sikand P: TRPV1: a target for next generation analgesics. Curr Neuropharmacol 6(2): 151-163, 2008. PMID: 19305794. DOI: 10.2174/157015908784533888

16 Caviedes-Bucheli J, Moreno JO, Ardila-Pinto J, Del ToroCarreno HR, Saltarín-Quintero H, Sierra-Tapias CL and Munoz HR: The effect of orthodontic forces on calcitonin gene-related peptide expression in human dental pulp. J Endod 37(7): 934937, 2011. PMID: 21689547.

17 Takahashi N, Matsuda Y, Sato K, De Jong PR, Bertin S and Tabeta K: Neuronal TRPV1 activation regulates alveolar bone resorption by suppressing osteoclastogenesis via CGRP. Sci Rep 6: 29294, 2016. PMID: 27388773. DOI: 10.1038/srep29294

18 Zhou Y, Long H, Ye N, Liao L, Yang X and Jian F: The effect of capsaicin on expression patterns of CGRP in trigeminal ganglion and trigeminal nucleus caudalis following experimental tooth movement in rats. J Appl Oral Sci 24(6): 597-606, 2016. PMID: 28076465. DOI: 10.1590/1678-775720160150

19 Alansari S, Atique MI, Gomez JP, Hamidaddin M, Thirumoorthy SN and Sangsuwon C: The effects of brief daily vibration on clear aligner orthodontic treatment. JWFO 7(4): 134-140, 2018. DOI: $10.1016 /$ j.ejwf.2018.10.002
20 Kraiwattanapong K and Samruajbenjakun B: Effects of different force magnitudes on corticotomy-assisted orthodontic tooth movement in rats. Angle Orthod 88(5): 632-637, 2018. DOI: 10.2319/103117-736.1

21 Yang Z, Luo W, Hou J, Zhao Z, Jian F and Wamalwa P: Development of a behavior model of pain induced by experimental tooth movement in rats. Eur J Oral Sci 117(4): 380-384, 2009. PMID: 19627348. DOI: 10.1111/j.1600-0722.2009.00639.x

22 Bae YC, Oh JM, Hwang SJ, Shigenaga Y and Valtschanoff JG: Expression of vanilloid receptor TRPV1 in the rat trigeminal sensory nuclei. J Comp Neurol 478(1): 62-71, 2004. PMID: 15334649. DOI: $10.1002 / \mathrm{cne} .20272$

23 Guo R, Zhou Y, Long H, Shan D, Wen J and Hu H: Transient receptor potential Vanilloid 1-based gene therapy alleviates orthodontic pain in rats. Int J Oral Sci 11(1): 11, 2019. PMID: 30853711. DOI: 10.1038/s41368-019-0044-3

24 Abràmoff MD, Magalhães PJ and Ram SJ: Image processing with ImageJ. Biophotonics Int 11(7): 36-42, 2004. DOI: 10.1038/nmeth.2089

25 Polat $\mathrm{O}$ and Karaman AI: Pain control during fixed orthodontic appliance therapy. Angle Orthod 75: 214-219, 2005. PMID: 15825785. DOI: 10.1043/0003-3219(2005)075<0210:PCDFOA> 2.0.CO;2

26 Gao Y, Liu Y, Zhu K, Zhang Z, Qiao H and Lu Z: Blocking of TRPV-1 in the parodontium relieves orthodontic pain by inhibiting the expression of TRPV-1 in the trigeminal ganglion during experimental tooth movement in rats. Neurosci Lett 628: 67-72, 2016. PMID: 27267133. DOI: 10.1016/j.neulet.2016. 06.007

27 Lundy F and Linden G: Neuropeptides and neurogenic mechanisms in oral and periodontal inflammation. Crit Rev Oral Biol Med 15(2): 82-98, 2004. PMID: 15059944. DOI: 10.1177/154411130401500203

28 de Hoon JN, Pickkers P, Smits P, Struijker-Boudier HA and Van Bortel LM: Calcitonin gene-related peptide: Exploring its vasodilating mechanism of action in humans. Clin Pharmacol Ther 73(4): 312-321, 2003. PMID: 12709721. DOI: 10.1016/ S0009-9236(03)00007-9 